\title{
Coherent cavitation in the liquid of light
}

\author{
Ángel Paredes, David Feijoo and Humberto Michinel \\ Área de Óptica, Departamento de Física Aplicada, \\ Universidade de Vigo, As Lagoas s/n, Ourense, ES-32004 Spain.
}

\begin{abstract}
We study the cubic (focusing)-quintic (defocusing) non-linear Schrödinger equation in two transverse dimensions. We discuss a family of stationary travelling waves, including rarefaction pulses and vortex-antivortex pairs, in a background of critical amplitude. We show that these rarefaction pulses can be generated inside a top-flat soliton when a smaller bright soliton collides with it. The fate of the evolution strongly depends on the relative phase of the solitons. Among several possibilities, we find that the dark pulse can re-emerge as a bright soliton.
\end{abstract}

PACS numbers: 42.65.Jx, 42.65.Tg, 05.45.Yv, 02.30.Jr

Introduction.- The cubic (focusing)-quintic (defocusing) non-linear Schrödinger equation (NLSE) reads:

$$
i \partial_{z} \psi=-\nabla_{T}^{2} \psi-\left(|\psi|^{2}-|\psi|^{4}\right) \psi,
$$

where $\nabla_{T}^{2}$ is the Laplacian in $d$ dimensions and the absolute values of the coefficients have been taken to unity without loss of generality. Eq. (1) is a paradigmatic model to describe fluids in which an attractive interaction becomes repulsive at short distances. It has been applied to superfluids [1], Bose-Einstein condensates 2], nuclear matter [3] or plasmas [4], among other fields [5]. In non-linear optics [6], $d=2$ when dispersion effects are negligible. Light beams acquire peculiar properties and can reach a phase with qualitative and quantitative similarities to a liquid, as theoretically discussed in [7]. Media with non-linear refractive indices resembling (11) include chalcogenide materials [8] among others [9]. Following the idea of [10] of using engineered coherent media, the experimental realization of this liquid of light has been achieved recently in a gas of sodium [11].

The liquid-like features are related to the existence of stable solitons and vortices [12] in $d=2$. For large $\int|\psi|^{2} d S$, these solitary waves become top-flat: there is a region where $\psi \approx \exp \left(i \beta_{c r} z\right) \Psi_{c r}$ and $\psi \approx 0$ elsewhere. This is reminiscent of instanton interpolation between vacua [34], where instanton action is identified with surface tension. The critical amplitude and propagation constant are [13]: $\Psi_{c r}=\sqrt{3} / 2, \beta_{c r}=3 / 16$.

In this Letter, we analyse travelling wave solutions in a background of critical amplitude. These bubbles generated and evolving within a fluid are a realization of the phenomenon of cavitation. In the liquid described by Eq. (1), cavitation can take place when flowing past an obstacle [14] or by explosion [15]. Alternatively, we discuss a situation in which the coherence properties of the NLSE allow interference, providing a scenario with essential differences to regular liquids.

We find a family of travelling waves of constant velocity [35] and shape, including rarefaction pulses (moving bubbles without vorticity) and vortex-antivortex pairs. These computations follow the seminal work [16] (see also [17]) for cubic NLSE and its generalizations [18, 19].
Then, we simulate collisions of a soliton of small power and size with a top-flat soliton. Different outcomes are possible, depending on their relative phase and speed: both solitons may merge in a single droplet, the impinging soliton may bounce back or a rarefaction pulse of the aforementioned family may be generated. This dark excitation sometimes re-emerges as a bright solitary wave.

In view of these qualitatively distinct results, it is conceivable to use the present set-up for interferometric purposes or optical switching. For instance, it may be useful to measure relative phases and speeds in experiments of the liquid of light as those described in [11], and to determine the coherence of the beams. This procedure to generate rarefaction pulses could find interesting applications such as studying their interaction with vortices [20], their stability properties when embedded in three dimensions [21] (with second order dispersion) or their dynamics in the presence of smooth inhomogeneities [22].

Travelling waves.- We look for stationary solutions of (1) moving at constant speed $U$ in the $x$-direction. In order to be form-preserving, $\psi$ takes the form $\psi(x, y, z)=$ $e^{i \beta_{c r} z} \Psi(x-U z, y)$ [16]. Defining a new coordinate $\eta=$ $x-U z$, the partial differential equation (PDE) (11) for $\psi(x, y, z)$ is reduced to a $\operatorname{PDE}$ for $\Psi(\eta, y)$ valid for any $z$. For instance we can take $z=0$ where $\eta=x$ and write:

$$
i U \partial_{x} \Psi=\nabla^{2} \Psi+\left(|\Psi|^{2}-|\Psi|^{4}-3 / 16\right) \Psi
$$

where $\nabla^{2}=\partial_{x}^{2}+\partial_{y}^{2}$. Boundary conditions are $\Psi \rightarrow$ $\Psi_{c r}=\sqrt{3} / 2$, as $r^{2}=x^{2}+y^{2} \rightarrow \infty$ in any direction. Momentum and energy are defined by:

$$
\begin{aligned}
p & =\frac{1}{2 i} \int\left[\left(\Psi^{*}-\Psi_{c r}\right) \partial_{x} \Psi-\left(\Psi-\Psi_{c r}\right) \partial_{x} \Psi^{*}\right] d x d y \\
E & =\int|\nabla \Psi|^{2} d x d y+\frac{1}{3} \int|\Psi|^{2}\left(|\Psi|^{2}-\Psi_{c r}^{2}\right)^{2} d x d y(3)
\end{aligned}
$$

If the derivative is taken over the family of solutions, $U=\partial E / \partial p$ holds. $\Psi$ is even under $y \rightarrow-y$ whereas its imaginary (real) part is odd (even) under $x \rightarrow-x$.

All solutions are subsonic $U<U_{0}$ where $U_{0}=\sqrt{3} / 2$ is the long-wavelength speed of sound. In the transonic limit $U \rightarrow U_{0}^{-}$, there is an explicit solution [16, 23]. We 
split real and imaginary parts as $\Psi=f+i g$ and introduce stretched coordinates $\xi=\epsilon^{2} y, \eta=\epsilon x$. Then:

$$
f=\Psi_{c r}+\epsilon^{2} f_{1}+\epsilon^{4} f_{2}+\ldots, \quad g=\epsilon g_{1}+\epsilon^{3} g_{2}+\ldots
$$

with $U=U_{0}+\epsilon^{2} U_{1}+\epsilon^{4} U_{2}+\ldots$. Expanding in $\epsilon$, one finds $f_{1}=-\frac{1}{\sqrt{3}} g_{1}^{2}+\frac{2}{\sqrt{3}} \partial_{\eta} g_{1}$ and a Kadomtsev-Petviashvili equation for $g_{1}$ :

$$
\frac{4 U_{1}}{\sqrt{3}} \frac{\partial^{2} g_{1}}{\partial \eta^{2}}-\frac{\partial^{2} g_{1}}{\partial \xi^{2}}+\frac{4}{3} \frac{\partial^{4} g_{1}}{\partial \eta^{4}}-16 \frac{\partial g_{1}}{\partial \eta} \frac{\partial^{2} g_{1}}{\partial \eta^{2}}=0
$$

which, for $U_{1}=-\sqrt{3} / 4$, is solved by:

$$
g_{1}=\frac{-2 \eta}{\eta^{2}+\xi^{2}+4}, \quad f_{1}=-\frac{4\left(4+\xi^{2}\right)}{\sqrt{3}\left(\eta^{2}+\xi^{2}+4\right)^{2}}
$$

The value of $U_{1}$ can be rescaled with $\epsilon$, but its sign is important since it shows that $U<U_{0}$ in this limit. This provides the low momentum limit of the family of travelling waves $E \approx \pi \epsilon, p=2 \pi \epsilon / \sqrt{3}$, which corresponds to rarefaction pulses. Solutions for $p \rightarrow \infty$, correspond to a well-separated vortex-antivortex pair with $U \rightarrow 0$.

We have computed numerical approximations to this family of solutions, following [19]: define a functional depending on a parameter $\mu=p+U$, such that solutions of (2) are its minimisers. Given a judicious initial ansatz for $\Psi$, they are computed by a heat flow in some auxiliary time coordinate, see [19] for details. We found a first solution for large $\mu$ by using a vortex-antivortex ansatz in the spirit of 24] and then used it as starting point to go over the family by a subsequent iteration $\mu \rightarrow \mu-\delta \mu$. We have cross-checked the numerical approximations got at the end of each heat flow by requiring that the following virial identities, analogous to [16], 25], are satisfied within a $3 \%$ in the finite difference scheme:

$$
\begin{aligned}
E & =p U+\int\left(|\Psi|^{2}-\Psi_{c r}^{2}\right)\left(\frac{\sqrt{3}}{4}\left(|\Psi|^{2}-\frac{1}{4}\right)\left(\Psi+\Psi^{*}\right)\right. \\
& \left.+-\frac{2}{3}|\Psi|^{4}\right) d x d y=2 \int\left|\partial_{x} \Psi\right|^{2} d x d y \\
p U & =\frac{2}{3} \int|\Psi|^{2}\left(|\Psi|^{2}-\Psi_{c r}^{2}\right)^{2} d x d y
\end{aligned}
$$

Four examples are displayed in Fig. 1] The dispersion relation is presented in Fig. 5 below. Arguments in [18] prove that these solutions are orbitally stable. The stability of the family in [16] was first analysed in [26].

Static $(U=0)$ bubble solutions in cubic-quintic media were found long ago [27], even if they are unstable [28]. The travelling waves presented here are not finite velocity generalizations of these bubbles (such a generalization in one-dimension was discussed in [29]). We have checked that the static bubbles only exist for $\beta>\beta_{c r}$ and therefore the boundary conditions used in this section do not match those of any quiescent bubble.

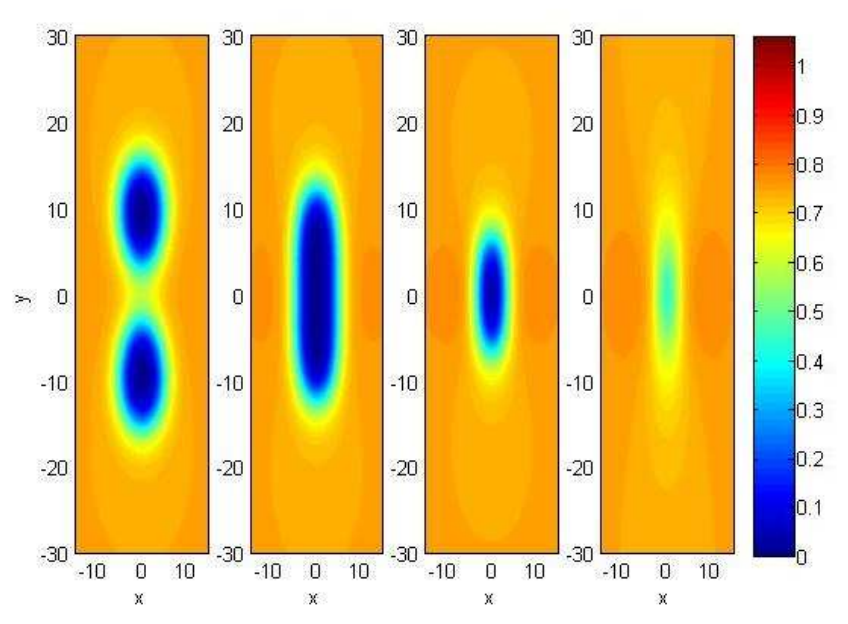

FIG. 1: (Color online) Contourmaps of $|\Psi|^{2}$ for four travelling waves. From left to right: $(U \approx 0.11, p \approx 80, E \approx 20.7)$; $(U \approx 0.21, p \approx 35.3, E \approx 12.5) ;(U \approx 0.35, p \approx 10.9$, $E \approx 6.1) ;(U \approx 0.71, p \approx 2.6, E \approx 2.1)$. The color scale also applies to Figs. 24].

Rarefaction pulses from soliton-soliton collisions.We now analyse the collision of two solitons of markedly different sizes by numerically solving Eq. (11). For these simulations, we use a standard split-step pseudo-spectral Fourier method - beam propagation method, see e.g [30] - in a lattice of $512 \times 256$ points. Even if it is a first order algorithm in $\Delta z$, we have used a fourth order Runge-Kutta to compute the evolution associated to the non-linear terms to avoid problems with high nonlinearities. Being an explicit method, it is only conditionally stable and a sufficiently small step $\Delta z$ must be taken, see [31] for more details on this issue. In order to write down the initial conditions, we notice that there exist smooth bright soliton solutions of the form $e^{i \beta_{s} z} \Psi_{s}(r)$ with $\beta_{s}<\beta_{c r}$ and $\lim _{r \rightarrow \infty} \Psi_{s}(r)=0$. Taking two of these and defining two radial coordinates with respect to each initial position, $r_{j}^{2}=\left(x-x_{j, 0}\right)^{2}+y^{2}$ for $j=1,2$ :

$$
\left.\psi\right|_{z=0}=\Psi_{s, 1}\left(r_{1}\right)+\Psi_{s, 2}\left(r_{2}\right) \exp \left(i \frac{v x}{2}+i \phi_{i}\right)
$$

corresponds to two separate solitons, the second one with initial velocity $v$. An initial relative phase $\phi_{i}$ has been included. Fixing the initial solitons and their positions, we can study the dynamics as a function of $v$ and $\phi_{i}$. As an illustrative example, in all the following plots we take $x_{1,0}=-22, x_{2,0}=130$ and solitons 1 and 2 to be those with $\beta_{s, 1} \approx 0.1856, \beta_{s, 2} \approx 0.15$, corresponding to total power $\int\left|\Psi_{s, 1}\right|^{2} d S \approx 3.1 \times 10^{4}, \int\left|\Psi_{s, 2}\right|^{2} d S \approx 86$ and radii $R_{s, 1} \approx 115, R_{s, 2} \approx 6.7$.

In Fig. 2, both droplets coalesce into one, and the impact results in the excitation of surface and body waves. In Fig. 3, a void is generated inside the droplet, which subsequently moves with constant velocity and eventually exits the top-flat soliton re-transformed in a bright 
soliton. This bright-dark-bright excitation conversion, reminiscent of the one-dimensional case [32], opens interesting possibilities. For fixed power of the initial solitons, the re-emergence of the small bright soliton only happens for certain ranges of initial soliton velocity and relative phase. Thus, it may be a useful probe to determine these parameters and/or the validity of the cubic-quintic model in a particular situation.
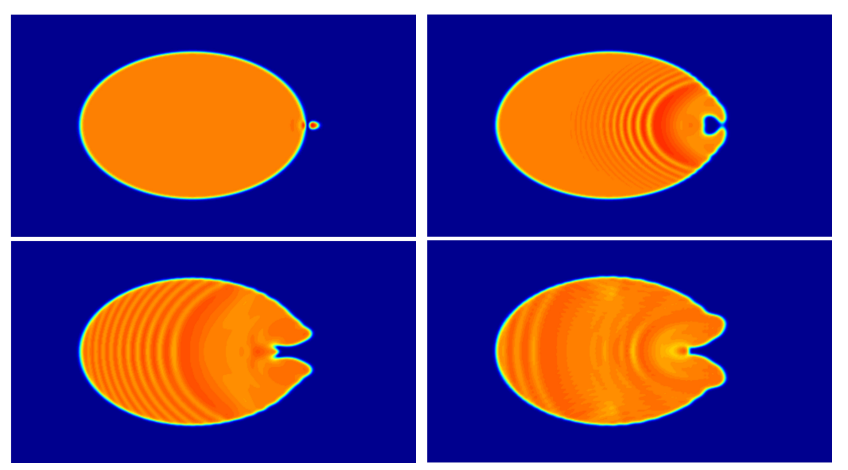

FIG. 2: (Color online) Evolution of $|\psi|^{2}$ with initial conditions $v=-1, \phi_{i}=\pi$. The four plots correspond to $z=30$, $z=90, z=150, z=210$, respectively. Here and in Fig. 3 . the horizontal axis is the $x$-direction, $x \in(-205,205)$ whereas the vertical axis is $y \in(-172,172)$.
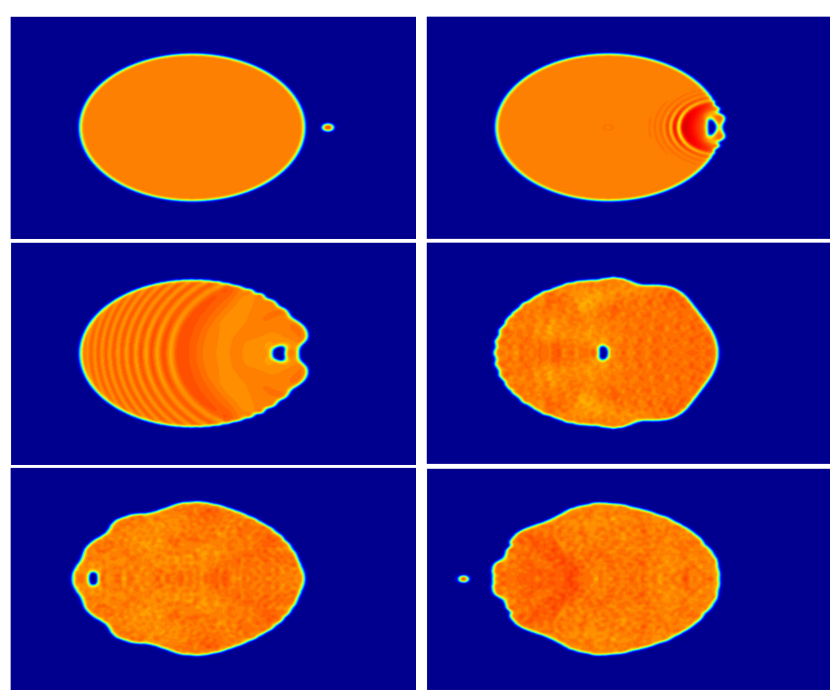

FIG. 3: (Color online) Evolution of $|\psi|^{2}$ with initial conditions $v=-1, \phi_{i}=0.7 \pi$. The six plots correspond to $z=15$, $z=60, z=150, z=525, z=900, z=1050$, respectively.

In Fig. 4, the evolution of $|\psi|^{2}(x, y=0)$ is plotted for the cases depicted above and for two other cases with different qualitative outcomes. On the bottom left, the small soliton bounces back - also in Fig. 2 part of the energy is reflected, although it is insufficient to form a bright soliton. On the bottom right, a fainter rarefaction pulse is formed but dies away when reaching the border of the top-flat soliton. Indeed, some rarefaction pulses propagate nearly undistorted for a distance but eventually fade away. This happens because the excitation is not exactly the stationary solution or because the medium is not infinite and constant. The pulse can vanish when reaching the border of the top-flat soliton or due to interaction with sound waves which also stem from the original collision. It can be appreciated that rarefaction pulses move with constant velocity $U$, always slower than sound waves (in Fig. 4, velocity is the angle with respect to the vertical axis). The relation between $U$ and $v$ is non-trivial: depending on the relative phase, the larger is the $E$ of the bubble, the smaller is its $U$.

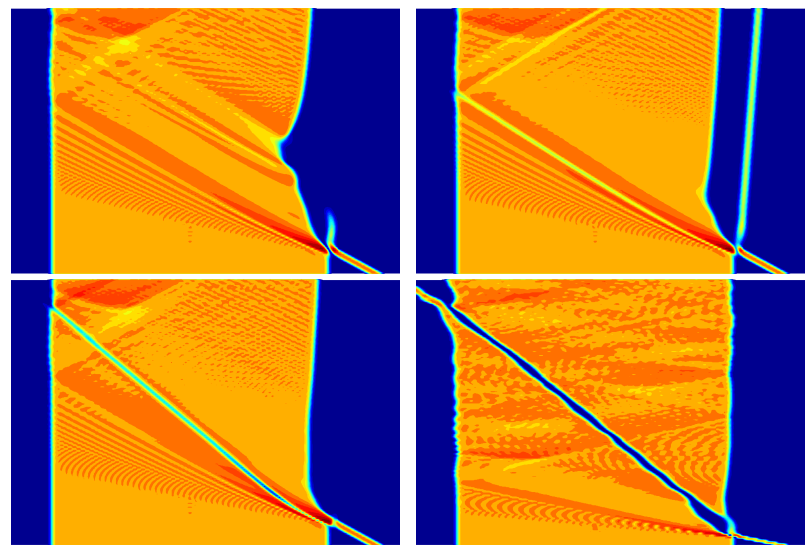

FIG. 4: (Color online) We depict the evolution in $z$ of $|\psi|^{2}(x, y=0)$ of the simulations of Figs. 2 (top left) and 3 (bottom right). The top right figure is for initial conditions $v=-1, \phi_{i}=1.3 \pi$ and the bottom left for $v=-1, \phi_{i}=1.6 \pi$ The horizontal axis corresponds to $x \in(-205,205)$. The vertical axis represents evolution from $z=0$ to $z=400$, except for the top right figure in which $z \in(0,1100)$.

Initial conditions of the aforementioned processes only differ in $\phi_{i}$. It is apparent that coherence plays an essential role in the phenomena discussed. Apart from perturbations qualitatively similar to those happening when droplets of regular liquids collide, there can be destructive interference near the surface of the large droplet. The non-linear interactions allow this disturbance to evolve into a stationary bubble, resembling a vapour cavity inside a liquid. In view of this analogy, we call this process coherent cavitation. To further elaborate on this point, we write the relative phase before the solitons meet:

$$
\phi_{\text {rel }}=-\phi_{i}-\left(\beta_{s, 2}-\beta_{s, 1}-\frac{v^{2}}{4}\right) z-\frac{v x}{2}
$$

Rarefaction pulses only appear above a limiting velocity $|v|>v_{\text {lim }}$. For the initial solitons of Figs. 2,4, $v_{\text {lim }} \approx 0.22$. The value of $v_{\text {lim }}$ is larger for smaller incoming solitons. For $|v|<v_{\text {lim }}$, the bounce is the most probable outcome. For $|v|>v_{\text {lim }}$, it is the formation of a rarefaction pulse, which therefore appears under fairly general initial conditions. The cases in which it is not generated can be understood, at least qualitatively, by 
considering Eq. (9). A rarefaction pulse appears unless $\phi_{\text {rel }}$ is around an integer multiple of $2 \pi$ at the collision. As an example, for the case of the figures, we have checked that the mentioned behaviour is observed in a considerable range of velocities $0.25 \lesssim|v| \lesssim 3.5$ by inserting in Eq. (9) $z=30.4 /|v|$ (where 30.4 is the initial soliton-soliton distance) and $x=90.6$, which is the position near the soliton edge where typically the larger $|\psi|^{2}$ disturbance is created after the collision (thus $\left.\phi_{\text {rel }} \approx 1.08 /|v|+52.9|v|-\phi_{i}\right)$.

In order to check that these bubbles in motion should be identified with the family of travelling waves found above (Fig. 11), we have performed a series of numerical experiments and compared their dispersion relation in Fig. 5. Eqs. (3) with two modifications were used to compute $p, E$. First, since the numerical integration gives $\psi$ rather than $\Psi$, we have introduced $\Psi=e^{-i \beta_{s, 1} z} \psi$. Second, the integration range cannot be taken to infinity. Our convention has been to perform the integral in a rectangle in the $x-y$ plane defined as follows: look for the positions $x_{l}, x_{r}$ where $|\psi|$ drops to $\Psi_{c r} / 2$ at $y=0$. The integration limits are $x_{l}-\Delta x<x<x_{r}+\Delta x$, $|y|<0.82\left(x_{r}-x_{l}\right)$, where we have fixed $\Delta x \approx 4$. This computation can be done for different values of $z$ for the same pulse. Results do not significantly differ as long as the rarefaction pulse is well within the large droplet - at least in a certain range of $z$. As expected [33], this dark solitonic excitation retains its identity even if the background has finite extent and is not perfectly stationary.

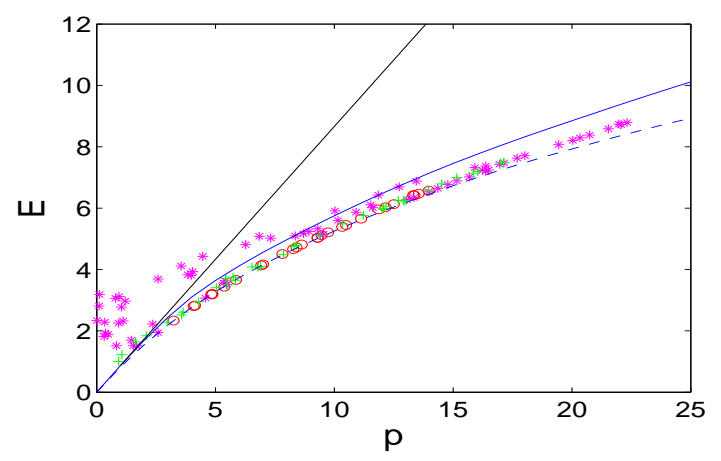

FIG. 5: (Color online) $E$ vs. $p$ for several rarefaction pulses generated with different values of $v$ and $\phi_{i}$ using three pairs of initial solitons - magenta asterisks for the case of Figs. 2, 4 ] green crosses for $\int\left|\Psi_{s, 1}\right|^{2} d S \approx 2.8 \times 10^{4}, \int\left|\Psi_{s, 2}\right|^{2} d S \approx 42$ and red circles for $\int\left|\Psi_{s, 1}\right|^{2} d S \approx 3.1 \times 10^{4}, \int\left|\Psi_{s, 2}\right|^{2} d S \approx 33$. The solid and dashed curves correspond to the family of travelling waves of Fig. 1 - for the dashed one the domain in the integrals (3) is cut as described above. The black solid line corresponds to sound speed $E=U_{0} p$.

Larger impinging solitons can generate larger bubbles (greater $p, E$ ). They also disturb more the top flat soliton, explaining why some magenta asterisks deviate from the stationary dispersion relation for small $p$.
Conclusion.- We have found noteworthy qualitative behaviours in the cubic-quintic NLSE, including rarefaction pulses generated by soliton-soliton interference. We have shown that these bubbles can be identified with a family of stationary solutions. In view of the numerous applications of the model (11) and the interest raised by the recent experiments [11], we hope that our results might inspire novel experiments of non-linear optics in atomic gases and, possibly, in other fields.

Acknowledgements.- We thank A. Ferrando, J. R. Salgueiro and D. Tommasini for discussions. The work of A. Paredes is supported by the Ramón y Cajal programme. The work of D. Feijoo is supported by the FPU Ph.D. programme. The work of A. Paredes and D. Feijoo is also supported by Xunta de Galicia through grant EM2013/002.

[1] V. L. Ginzburg and A. A. Sobyanin, J. Low Temp. Phys. 49, 507 (1982). C. Josserand and S. Rica, Phys. Rev. Lett. 78, 1215 (1997).

[2] A. Bulgac, Phys. Rev. Lett. 89, 050402 (2002). F. Kh. Abdullaev, A. Gammal, L. Tomio, and T. Frederico, Phys. Rev. A 63, 043604 (2001). A. Muryshev, G.V. Shlyapnikov, W. Ertmer, K. Sengstock, and M. Lewenstein, Phys. Rev. Lett 89110401 (2002). L. Khaykovich and B.A. Malomed, Phys. Rev. A 74, 023607 (2006). A.-X. Zhang and J.-K. Xue, Phys. Rev. A 75, 013624 (2007). R. Carretero-González, D.J. Frantzeskakis and P.G. Kevrekidis, Nonlinearity 21 (2008) R139-R202. B.B. Baizakov, A. Bouketir, A. Messikh, A. Benseghir, B.A. Umarov, Int. Jour. Mod. Phys. B 25, 2427-2440 (2011). C. Trallero-Giner, R. Cipolatti, T.C. H. Liew, Eur. Phys. J. B 67, 143 (2013).

[3] V.G. Kartavenko, Sov. J. Nucl. Phys. 40 (1984) 240. V. G. Kartavenko, A. Sandulescu, W. Greiner, Int. J. Mod. Phys. E 7 (1998) 449-463.

[4] C. Zhou and X. T. He, Phys. Scr. 50 ,415-418 (1994). T. A. Davydova, A. I. Yakimenko, and Yu. A. Zaliznyak, Phys. Rev. E 67, 026402 (2003).

[5] I.V. Barashenkov, E.Y. Panova, Physica D 69 (1993) 114-134. Song Xiang, Li Hua-Mei, Commun. Theor. Phys. 59 (2013) 290294.

[6] D. Mihalache, D. Mazilu, M. Bertolotti, C. Sibilia, J. Opt. Soc. Am. B 5, 565 (1988). D. Pushkarov, S. Tanev, Opt. Comm. 124 (1996), 354-364. K. Dimitrevski et al., Phys. Lett. A 248, 369 (1998).

[7] D. E. Edmundson and R. H. Enns, Phys. Rev. A 51, 2491 (1995). M. Quiroga-Teixeiro and H. Michinel, J. Opt. Soc. Am. B 14, 2004 (1997). D. Mihalache et al., Phys. Rev. Lett. 88, 073902 (2002). H. Michinel, J. CampoTáboas, R. García-Fernández, J. R. Salgueiro, and M. L. Quiroga-Teixeiro, Phys. Rev. E 65, 066604 (2002). M. J. Paz-Alonso, D. Olivieri, H. Michinel, and J. R. Salgueiro, Phys. Rev. E 69, 056601 (2004). D. Novoa, H. Michinel, D. Tommasini, Phys. Rev. Lett. 103, 023903 (2009).

[8] F. Smektala, C. Quemard, V. Couderc, and A. Barthelemy, J. Non-Cryst. Solids 274, 232 (2000).

[9] R.M. Caplan, R. Carretero-Gonzalez, R. Kevrekidis, 
B.A. Malomed, Math. Comp. Simul. 82, 1150-1171 (2012).

[10] H. Michinel, M. J. Paz-Alonso, V. M. Pérez-García, Phys. Rev. Lett 96, 023903 (2006). A. Alexandrescu, H. Michinel, V. M. Pérez-García, Phys. Rev. A 79, 013833 (2009).

[11] Z. Wu, Y. Zhang, C. Yuan, F. Wen, H. Zheng, Y. Zhang, M. Xiao, Phys. Rev. A 88, 063828 (2013).

[12] M. Quiroga-Teixeiro, H. Michinel, J. Opt. Soc. Am. B 14 (1997) 20042009. I. Towers, A.V. Buryak, R.A. Sammut, B.A. Malomed, L.-C. Crasovan, Dumitru Mihalache, Phys. Lett. A 292, 288 (2001). B.A. Malomed, L.-C. Crasovan, D. Mihalache, Physica D 161 (2002) 187201. H. Michinel, J.R. Salgueiro, M.J. Paz-Alonso, Phys. Rev. E 70, 066605 (2004).

[13] V. Prytula, V. Vekslerchik, and V. M. Pérez-García, Phys. Rev. E 78, 027601 (2008).

[14] C. Josserand, Y. Pomeau, S. Rica, Phys. Rev. Lett. 75, 3150-3154 (1995).

[15] C. Josserand, Phys. Rev. E 60, 482-491 (1999).

[16] C.A. Jones, P.H. Roberts, J. Phys. A: Math. Gen. 15 (1982), 2599-2619.

[17] F. Béthuel, P. Gravejat and J-C. Saut, Comm. Math. Phys. 285 567-651 (2009).

[18] D. Chiron, M. Maris, preprint arXiv:1203.1912 [math.AP] (2012).

[19] D. Chiron, C. Scheid, preprint HAL-Inria Open Archive hal-00873794 (2013).

[20] N.G. Berloff, Phys. Rev. A 69, 053601 (2004).

[21] N.G. Berloff, Phys. Rev. B 65, 174518 (2002).

[22] L.A. Smirnov, V.A. Mironov, Phys. Rev. A 85, 053620 (2012).
[23] F. Béthuel, P. Gravejat and J-C. Saut, Dynamics of PDE 5, 3 (2008), 241-280. D. Chiron, M. Maris, preprint arXiv:1210.1315 1 [math.AP] (2012).

[24] N.G. Berloff, J. Phys. A: Math. Gen. 37 (2004), 16171632.

[25] C.A. Jones, S.J. Putterman, P.H. Roberts, J. Phys. A: Math. Gen. 19 (1986), 2991-3011.

[26] N.G. Berloff and P.H. Roberts, J. Phys. A: Math. Gen. 37 (2004) 1133311351.

[27] I.V. Barashenkov, V.G. Makhankov, Phys. Lett A 128, 52-56 (1988).

[28] I.V. Barashenkov, A.D. Gocheva, V.G. Makhankov, I.V. Puzynin, Physica D 34 (1989), 240-254.

[29] I.V. Barashenkov, T.L. Boyadjiev, I.V. Puzynin, T. Zhanlav, Phys. Lett. A 135, 125-128 (1989).

[30] G.P. Agrawal, Nonlinear Fiber Optics 4th Ed., Elsevier/Academic Press, 2006. T.-C. Poon, T. Kim, Engineering Optics with Matlab, World Scientific, Singapore, 2006.

[31] J.A.C. Weideman, B.M. Herbst, Siam J. Numer. Anal., 23, 485 (1986). T. I. Lakoba, Numer. Meth. Part. D. E., 28, 641 (2010).

[32] W. Kim, H.-T. Moon, Phys. Lett. A 266, 364-369 (2000). W. Kim, H.-T. Moon, J. Korean Phys. Soc. 38, 558-561 (2001).

[33] Y.S. Kivshar, B. Luther-Davies, Phys. Rep. 298 (1998), 81-197.

[34] We thank Albert Ferrando for suggesting this analogy.

[35] We loosely use the term velocity even if the coordinate associated to evolution of the NLSE is propagation distance rather than time. 